\title{
Resultados y complicaciones de la enfermedad del segmento adyacente tratada mediante artrodesis intersomática lateral mínimamente invasiva
}

\author{
Results and complications of adjacent segment disease treated by \\ minimally invasive lateral intersomatic arthrodesis \\ Luque R,* Echevarría M,* Alcobía B,* Urda A,* Domínguez I,* Marco F* \\ Hospital Clínico San Carlos Madrid.
}

RESUMEN. Introducción: Se ha realizado un estudio clínico comparativo sobre los pacientes intervenidos mediante artrodesis intersomática lateral para tratamiento de la enfermedad del segmento adyacente utilizando dispositivos intersomáticos de titanio y de PEEK. Material y métodos: Se han analizado y comparado los resultados clínicos (EVA y oswestry disability index ODI) y radiológicos (alineamiento y fusión), las complicaciones (mayores y menores) y la calidad de vida (EQ5D) de 32 pacientes intervenidos desde Septiembre de 2015 hasta Septiembre de 2018, con un seguimiento medio de 25 meses (46-18). La edad media en la cirugía fue de 66 años (39-89) y 68\% de los pacientes fueron mujeres. El segmento intervenido con más frecuencia fue L3-L4 (62\%) abordaje retroperitoneal derecho 86\%. La EVA lumbar mejoró de $6.2 \pm 2.12$ a $4.1 \pm 1.71(\mathrm{p}=0.028)$. La EVA de la pierna descendió de $5.3 \pm 2.26$ a $1.9 \pm 1.58(\mathrm{p}=0.02)$. La escala ODI mejoró de $50.2 \pm 18.9$ a $33.3 \pm 10.2(\mathrm{p}=0.025)$ y la EQ5D pasó de 0.52 a $0.73(\mathrm{p}=0.039)$ sin diferencias estadísticamente significativas entre los grupos (ODI $\mathrm{p}=0.18, \mathrm{EQ} 5 \mathrm{D} \mathrm{p}=$ 0.293). Radiológicamente aumentó la altura intervertebral, la lordosis lumbar y segmentaria, disminuyó el ángulo de Cobb y la tasa de fusión global fue de 84.3\% (88\% Ti/82\% PEEK), sin diferencias entre los grupos. Conclusiones: La artrodesis intersomática lumbar lateral Lateral Lumbar Interbody Fusion es un método eficaz para el tratamiento de la enfermedad del segmento adyacente con resultados clínicos-radiológicos y complicaciones similares a la literatura. No se han encontrado diferencias entre los implantes de Ti y de PEEK.
ABSTRACT. Introduction: A comparative clinical study has been conducted on patients involved using lateral intersomatic arthrodesis for the treatment of adjacent segment disease using titanium and PEEK intersomatic devices. Material and methods: Clinical (EVA and oswestry disability index ODI) and radiological (alignment and fusion), complications (major and minor) and quality of life (EQ5D) of 32 patients intervened from September 2015 to September 2018 have been analyzed and compared, with an average follow-up of 25 months (46-18). The average age in surgery was 66 years (3989 ) and $68 \%$ of patients were women. Results: The most common segment involved was L3-L4 (62\%) right retroperitoneal approach $86 \%$. Lumbar EVA improved from $6.2 \pm 2.12$ to $4.1 \pm 1.71(\mathrm{p}=0.028)$. The LEG EVA descended from $5.3 \pm 2.26$ to $1.9 \pm 1.58(\mathrm{p}=0.02)$. The ODI scales improved from $50.2 \pm 18.9$ to $33.3 \pm 10.2(\mathrm{p}=$ $0.025)$ and the EQ5D went from 0.52 to 0.73 ( $p=0.039)$ with no statistically significant differences between the groups (ODI p $=0.18$, EQ5D p = 0.293). Radiologically increased intervertebral height, lumbar and segmental lordosis, decreased Cobb's angle and the overall melting rate was $84.3 \%$ (88\% Ti/82\% PEEK), with no differences between the groups. Conclusion: Lateral lumbar interbody fusion is an effective method for treating adjacent segment disease with clinical-radiological results and literature-like complications. No differences have been found between Ti and PEEK implants.

\section{Nivel de evidencia: III. Estudio retrospectivo comparativo}

* Unidad de Columna. Servicio de Cirugía Ortopédica y Traumatología. Hospital Clínico San Carlos Madrid.

Correspondencia:

Rafael Luque

Unidad de Columna. Servicio de Cirugía Ortopédica y Traumatología, Hospital Clínico San Carlos Madrid.

E-mail: doctorluque@yahoo.es

Citar como: Luque R, Echevarría M, Alcobía B, Urda A, Domínguez I, Marco F. Resultados y complicaciones de la enfermedad del segmento adyacente tratada mediante artrodesis intersomática lateral mínimamente invasiva. Acta Ortop Mex. 2020; 34(6): 388-398. https://dx.doi.org/10.35366/99137

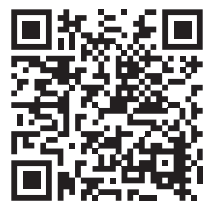


Palabras clave: Segmento adyacente, lateral, columna, cirugía, artrodesis.
Keywords: Adjacent segment, lateral, spine, surgery, arthrodesis.

\section{Introducción}

La fusión vertebral lumbar es un procedimiento en continuo auge, se estima que aproximadamente se realizan alrededor de 400,000 intervenciones anuales en Estados Unidos. ${ }^{1,2}$ A los cinco años de una cirugía de artrodesis lumbar hasta $40 \%$ de los pacientes comienzan a desarrollar cambios radiológicos de enfermedad del segmento adyacente (ESA) y casi la mitad de estos pacientes volverán a consulta de cirugía de columna por empeoramiento clínico. ${ }^{3,4,5,6}$ Un porcentaje importante será reintervenido por aumento de dolor radicular, claudicación neurógena o dolor mecánico lumbar. ${ }^{7,8}$ Según la progresión de la ESA, será necesario la descompresión (laminectomía, foraminotomía, facectomía) y/o extensión de la fusión vertebral (anterior (ALIF), transforaminal (TLIF) o posterior (PLIF)). . $^{3,8,9,10,11}$ La cirugía de revisión presenta una alta tasa de complicaciones debidas a los tiempos quirúrgicos prolongados, a las grandes pérdidas hemáticas y a las largas estancias hospitalarias con un riesgo de lesión neurológica (lesión del saco dural) ${ }^{12,13}$ y con tasas de reintervención que oscilan alrededor de $25 \% .^{14}$

Las publicaciones acerca del tratamiento de la ESA que utiliza el abordaje transpoas mínimamente invasivo para realizar la fusión lateral (LLIF) presentan menor porcentaje de complicaciones y resultados similares en cuanto a tasa de artrodesis y mejoría clínica. Con esta cirugía se obtiene una fusión vertebral anterior y una descompresión indirecta de los elementos neurológicos con menor daño en las partes blandas, tiempo quirúrgico más corto y movilización precoz del paciente..$^{15,16}$

Un aspecto clave en el tratamiento de la ESA es la realización de una fusión intersomática, con ella se busca la estabilidad de la columna anterior, la mejoría en el perfil sagital lumbar y una descompresión indirecta neural por distracción de las partes blandas. ${ }^{17,18,19}$ Para conseguir la fu- sión vertebral se utilizan implantes metálicos de titanio (Ti) desde 1940 e implantes plásticos de Poly-ether-ether-ketone (PEEK) desde $1990 .{ }^{20}$ Se han publicado múltiples estudios que evalúan los resultados de estos implantes a pesar de lo cual no hay consenso en cuanto a su uso. ${ }^{21,22}$ Por todos estos motivos hemos realizado un estudio clínico de los pacientes intervenidos mediante LLIF para tratamiento de la ESA utilizando dispositivos intersomáticos de Ti y de PEEK.

\section{Material y métodos}

Se han analizado y comparado los resultados clínicos y radiológicos, las complicaciones y la calidad de vida de un total de 32 pacientes intervenidos desde Septiembre de 2015 hasta Septiembre de 2018, con un seguimiento medio de 25 meses (máximo 46 meses y mínimo 18 meses).

Se recogieron las características médicas, situación clínica preoperatoria (escala visual analógica (EVA) lumbar y ciático; Owestry Disability Score (ODI)), la calidad de vida (EuroQol-5D (EQ5D)) y los parámetros radiológicos prequirúrgicos (lordosis lumbar (LL): medición angulación sagital desde el platillo superior L1 a platillo inferior L5; lordosis nivel (LN): medición angulación sagital desde el platillo superior vértebra superior a platillo inferior de la vértebra inferior; Cobb nivel $(\mathrm{CN})$ : angulación frontal platillo superior vértebra superior a platillo inferior de la vértebra inferior; y la altura del disco intervenido (ADI): platillo inferior vértebra superior a platillo superior de la vértebra inferior (medido en radiología anteroposterior y lateral) de todos los pacientes. La edad media en el momento de la cirugía fue de 66 años (39-89). Del total de pacientes, 68\% fueron mujeres. La patología médica más frecuente fue la HTA (21\%). Todos los pacientes presentaban como patología a tratar enfermedad del segmento adyacente: clínicamente sufrían de dolor lumbar crónico e irradiado por miembros inferiores
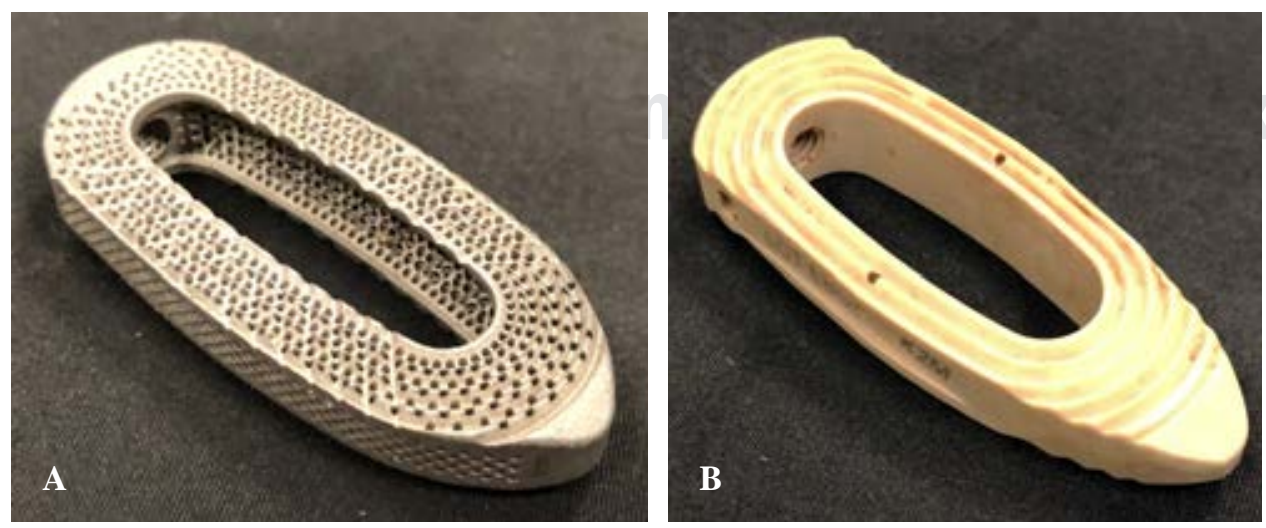

Figura 1:

Imágenes de los dispositivos intersomáticos utilizados, A) El Implante de titanio y B) El de PEEK. PEEK = poly-ether-ether-ketone. 


\begin{tabular}{|c|c|c|c|c|}
\hline & $\begin{array}{c}\text { General } \\
(\mathrm{N}=29) \mathrm{n}(\%)\end{array}$ & $\begin{array}{l}\text { PEEK } \\
(\mathrm{n}=16)\end{array}$ & $\begin{array}{l}\text { Titanio } \\
(\mathrm{n}=13)\end{array}$ & $\mathrm{p}$ \\
\hline Edad & $66(39-85)$ & $64(39-85)$ & $68(47-80)$ & 0.541 \\
\hline \multicolumn{5}{|l|}{ Sexo } \\
\hline Mujeres & $18(68)$ & 10 & 8 & 0.871 \\
\hline Varones & $11(32)$ & 7 & 4 & \\
\hline \multicolumn{5}{|l|}{ Fumador } \\
\hline Sí & $14(47)$ & 8 & 6 & 0.737 \\
\hline No & $12(53)$ & 6 & 6 & \\
\hline \multicolumn{5}{|l|}{ Patología endocrina } \\
\hline Diabetes mellitus & $4(16)$ & 3 & 1 & 0.362 \\
\hline Obesidad & $2(11)$ & 1 & 1 & 0.368 \\
\hline Dislipidemia & $8(32)$ & 4 & 4 & 0.376 \\
\hline Hipotiroidismo & $4(16)$ & 3 & 1 & 0.386 \\
\hline \multicolumn{5}{|l|}{ Patología cardíaca } \\
\hline HTA & $6(21)$ & 3 & 3 & 0.073 \\
\hline ICC & $1(5)$ & 0 & 1 & 0.98 \\
\hline FA & $1(5)$ & 1 & 0 & 0.063 \\
\hline Valvulopatía & $1(5)$ & 1 & 0 & 0.187 \\
\hline \multicolumn{5}{|l|}{ Patología pulmonar } \\
\hline EPOC & $1(5)$ & 1 & 0 & 0.023 \\
\hline \multicolumn{5}{|l|}{$\begin{array}{l}\text { Patología neuro- } \\
\text { lógica }\end{array}$} \\
\hline AIT & $1(5)$ & 1 & 1 & 0.020 \\
\hline Vértigos & $1(5)$ & 1 & 0 & \\
\hline \multicolumn{5}{|l|}{ Patología digestiva } \\
\hline Gastritis crónica & $1(5)$ & 0 & 1 & 0.030 \\
\hline \multicolumn{5}{|l|}{ Patología infecciosa } \\
\hline VIH & $1(5)$ & 1 & 0 & 0.025 \\
\hline \multicolumn{5}{|l|}{$\begin{array}{l}\text { Patología } \\
\text { gine-urológica }\end{array}$} \\
\hline $\begin{array}{l}\text { Patología } \\
\text { prostática }\end{array}$ & $3(11)$ & 2 & 1 & \\
\hline Patología uterina & $3(11)$ & 1 & 2 & \\
\hline \multicolumn{5}{|l|}{ Patología siquiátrica } \\
\hline Depresión & $6(21)$ & 3 & 3 & \\
\hline EVA lumbar & $6.2 \pm 2.12$ & $6.1 \pm 1.78$ & $6.4 \pm 2.02$ & \\
\hline EVA pierna & $5.3 \pm 2.26$ & $5.1 \pm 2.26$ & $5.5 \pm 1.65$ & \\
\hline ODI & $50.2 \pm 18.9$ & $50.9 \pm 11.32$ & $48.5 \pm 17.4$ & 0.042 \\
\hline EQ5D & 0.52 & 0.51 & 0.57 & 0.021 \\
\hline $\begin{array}{l}\text { PEEK = poly-ether-ether } \\
\text { cardíaca crónica, FA = fi } \\
\text { obstructiva crónica, AIT } \\
\text { inmunodeficiencia huma }\end{array}$ & $\begin{array}{l}\text { etone, } \mathrm{HTA}=\text { hipe } \\
\text { llación auricular, } \mathrm{E} \\
\text { ataque isquémico t } \\
\text {, ODI = Oswestry }\end{array}$ & $\begin{array}{l}\text { rtensión arteria } \\
\text { POC = enferme } \\
\text { transitorio, VIH } \\
\text { Disability Inde }\end{array}$ & $\begin{array}{l}\text { al, ICC = insufici } \\
\text { edad pulmonar } \\
\text { = virus de la } \\
\text { ox. EO5D = Euro }\end{array}$ & iencia \\
\hline
\end{tabular}

con una discopatía lumbar con artrosis facetaria que condiciona estenosis foraminal proximal a una instrumentación lumbar previa, sin mejoría clínica tras terapia médica, física y rehabilitadora durante un período mínimo de seis meses.

\section{Técnica quirúrgica}

Las cirugías se han realizado con el paciente colocado en decúbito lateral, en mesa radiotransparente y quebrada en la zona lumbosacra. Todas las intervenciones se efectuaron con monitorización por neurofisiólogo. Se han utilizado cuatro técnicas de control neurofisiológico intraoperatorio: potenciales evocados motores tras estímulo transcraneal (TcMP), potenciales evocados somatosensoriales (SSEP),
EMG-libre (Free Running-EMG) y EMG-estimulado (Triggered-EMG). Mediante este control neurofisiológico todos los pasos quirúrgicos son controlados y mediante la detección de cambios en el registro y la modificación de la técnica para recuperar la actividad fisiológica pueden evitarse las complicaciones derivadas de esta técnica quirúrgica. En los casos en los que la anatomía de la columna lumbosacra, de los grandes vasos y de la pelvis nos lo permitió, posicionamos al paciente con el lado doloroso hacia arriba, es decir, el abordaje fue por el mismo lado donde se presentaba la clínica radicular. Con ayuda del intensificador de imágenes se realizó la localización del nivel a intervenir y se hizo una incisión de aproximadamente $3 \mathrm{~cm}$. Tras la piel y el tejido celular subcutáneo se abrió la fascia de la musculatura oblicua abdominal hasta el retroperitoneo. Mediante disección roma con el dedo se identificó la apófisis transversa de la vértebra superior, el músculo psoas y el disco intervertebral. Bajo control radioscópico se colocó la aguja guía en la posición adecuada y se hizo un primer control neurofisiológico. Mediante una serie de dilatadores se obtuvo una ventana de trabajo a través del músculo psoas. Este gesto se realizó siempre bajo un estricto control neurofisiológico intraoperatorio. El separador se introdujo a través de la aguja situada en el disco intervertebral y se inició su apertura. Bajo control radioscópico se localizó el lugar para fijar las valvas en los cuerpos vertebrales. Una vez localizado el disco intervertebral se abrió con el bisturí y se realizó la discectomía. Se procedió a preparar los platillos vertebrales hasta hueso subcondral y a introducir dilatadores que nos dieron una idea del tamaño, longitud y anchura del implante. Una vez decididas las medidas se introduce una caja de prueba y se comprueba su colocación bajo control radioscópico así como la estabilidad primaria de la misma.

\begin{tabular}{|c|c|c|c|c|}
\hline & $\begin{array}{l}\text { General } \\
(\mathrm{N}=29)\end{array}$ & $\begin{array}{c}\text { PEEK } \\
(\mathrm{n}=16)\end{array}$ & $\begin{array}{l}\text { Titanio } \\
(\mathrm{n}=13)\end{array}$ & $\mathrm{p}$ \\
\hline Menores & 12 & 6 & 6 & \\
\hline \multicolumn{5}{|l|}{ Sistémicas } \\
\hline Anemización & 7 & 3 & 4 & 0.618 \\
\hline Íleo paralítico & 3 & 2 & 1 & \\
\hline ITU & 2 & 1 & 1 & \\
\hline Hernia abdominal & 0 & 0 & 0 & \\
\hline \multicolumn{5}{|l|}{ Locales } \\
\hline Celulitis herida & 1 & 1 & 0 & 0.129 \\
\hline Plexopatía femoral & 1 & 1 & 0 & 0.129 \\
\hline Mayores & 1 & 1 & 0 & 0.129 \\
\hline \multicolumn{5}{|l|}{ Sistémicas } \\
\hline Exitus & 0 & 0 & 0 & \\
\hline Lesión radicular & 0 & 0 & 0 & \\
\hline Lesión vascular & 0 & 0 & 0 & \\
\hline Hematoma retroperitoneal & 0 & 0 & 0 & \\
\hline \multicolumn{5}{|l|}{ Locales } \\
\hline Infección profunda & 0 & 0 & 0 & \\
\hline
\end{tabular}

PEEK = poly-ether-ether-ketone, $\mathrm{ITU}=$ infección del tracto urinario. 
Tabla 3: Distribución de variables radiológicas (preoperatorio, uno, seis y 12 meses).

\begin{tabular}{|c|c|c|c|c|c|}
\hline \multicolumn{3}{|c|}{ Evolución parámetros radiológicos } & \multicolumn{3}{|c|}{ Comparación por grupos } \\
\hline & Media (mm) & $\begin{array}{l}\text { Significación } \\
\text { evolución }\end{array}$ & PEEK & Titanio & $\begin{array}{l}\text { Significación } \\
\text { comparación }\end{array}$ \\
\hline \multicolumn{6}{|c|}{ Altura disco AP (meses) } \\
\hline Pre & $7.61 \pm 3.31$ & & $6.50 \pm 3.5$ & $9.41 \pm 1.84$ & 0.048 \\
\hline 1 & $12.81 \pm 2.36$ & 0.000 & $12.36 \pm 2.55$ & $13.56 \pm 1.95$ & 0.269 \\
\hline 6 & $11.28 \pm 2.92$ & 0.006 & $10.24 \pm 2.61$ & $12.72 \pm 2.84$ & 0.065 \\
\hline 12 & $11.825 \pm 2.25$ & 0.023 & $10.88 \pm 1.28$ & $13.40 \pm 2.74$ & 0.024 \\
\hline \multicolumn{6}{|c|}{ Altura disco LAT } \\
\hline Pre & $7.64 \pm 3.55$ & & $6.61 \pm 4.01$ & $9.41 \pm 1.81$ & 0.091 \\
\hline 1 & $13.47 \pm 2.05$ & 0.001 & $13.18 \pm 2.31$ & $13.96 \pm 1.59$ & 0.410 \\
\hline 6 & $11.41 \pm 2.70$ & 0.010 & $10.66 \pm 2.33$ & $12.45 \pm 2.74$ & 0.159 \\
\hline 12 & $11.82 \pm 3.28$ & 0.010 & $10.73 \pm 2.11$ & $13.65 \pm 4.23$ & 0.084 \\
\hline \multicolumn{6}{|c|}{ Lordosis lumbar } \\
\hline Pre & $28.58 \pm 10.14$ & & $28.12 \pm 9.70$ & $29.32 \pm 11.42$ & 0.800 \\
\hline 1 & $32.619 \pm 8.83$ & 0.000 & $33.96 \pm 8.95$ & $30.44 \pm 8.75$ & 0.388 \\
\hline 6 & $33.13 \pm 10.30$ & 0.000 & $32.42 \pm 11.40$ & $34.11 \pm 9.24$ & 0.734 \\
\hline 12 & $31.81 \pm 8.925$ & 0.000 & $33.66 \pm 10.20$ & $29.44 \pm 6.99$ & 0.367 \\
\hline \multicolumn{6}{|c|}{ Lordosis segmentaria } \\
\hline Pre & $5.88 \pm 4.8$ & & $5.892 \pm 3.87$ & $5.863 \pm 3,02$ & 0.985 \\
\hline 1 & $10.74 \pm 4.12$ & 0.014 & $11.13 \pm 4.60$ & $10.11 \pm 3.2$ & 0.596 \\
\hline 6 & $10.41 \pm 2.70$ & 0.010 & $10.78 \pm 1.1$ & $9.85 \pm 2.1$ & 0.747 \\
\hline 12 & $9.82 \pm 3.28$ & 0.023 & $10.31 \pm 4.5$ & $9.71 \pm 1.1$ & 0.311 \\
\hline \multicolumn{6}{|l|}{ Cobb } \\
\hline Pre & $7.57 \pm 3.50$ & & $7.29 \pm 2.20$ & $8.01 \pm 5.10$ & 0.683 \\
\hline 1 & $4.61 \pm 2.63$ & 0.000 & $4.24 \pm 1.76$ & $5.20 \pm 3.70$ & 0.465 \\
\hline 6 & $4.20 \pm 3.64$ & 0.000 & $3.60 \pm 1.55$ & $5.30 \pm 5.95$ & 0.523 \\
\hline 12 & $3.90 \pm 3.31$ & 0.000 & $3.40 \pm 1.77$ & $4.70 \pm 5.12$ & 0.474 \\
\hline
\end{tabular}

El tipo del dispositivo (PEEK o titanio) fue elegido a criterio del cirujano. La caja se introdujo rellenada de matriz ósea desmineralizada (MOD) para facilitar la artrodesis intersomática (Figura 1). Los implantes utilizados tienen una altura desde $8 \mathrm{~mm}$ con incrementos de 2 en $2 \mathrm{~mm}$ hasta $14 \mathrm{~mm}$; una longitud desde $45 \mathrm{~mm}$ con incrementos de 5 en $5 \mathrm{~mm}$ hasta $60 \mathrm{~mm}$ y una anchura de 18 y $22 \mathrm{~mm}$. La caja intersomática se implantó con placa lateral atornillada, en los casos en los que la anatomía no lo permitió (por encontrarse el nervio femoral sobre la zona de trabajo) la caja se colocó stand-alone.

\section{Período postquirúrgico}

Se autorizó la marcha al día siguiente de la cirugía. Todos los pacientes utilizaron corsé termoplástico rígido a medida durante tres meses. La limitación de actividades físicas (laborales o deportivas) se mantuvo durante cuatro meses. No se autorizó hacer esfuerzos intensos hasta los seis meses de la intervención.

Las variables postquirúrgicas (resultados clínicos, de calidad de vida y los parámetros radiológicos) se recogieron tras la cirugía al primer, sexto y décimo segundo mes. Se realizó TC lumbar al año de la inntervención en búsqueda de tasa fusión, distinguiendo: fusión completa (formación de puentes óseos a través y alrededor del implante), fusión incompleta (puentes óseos a través o alrededor del implan- te) y falta de fusión (seudoartrosis) (Figura 2). ${ }^{23}$ Se registraron todas las complicaciones locales y sistémicas, intraoperatorias y postoperatorias durante el período hospitalario y durante el seguimiento. A su vez en cada grupo se distinguieron las complicaciones menores (recibieron tratamiento médico para su curación) y mayores (precisaron tratamiento quirúrgico para su resolución).

\section{Métodos estadísticos}

Se ha realizado un estudio estadístico comparativo de todos los parámetros recogidos analizando los resultados de la serie y de cada grupo comparándolos en búsqueda de diferencias. Para ello se ha llevado a cabo un estudio comparativo mediante SPSS 17.0 (SPSS Inc. Chicago USA). Las variables cualitativas se presentarán con su distribución de frecuencias e intervalo de confianza al $95 \%$ y las variables cuantitativas se resumieron con su media y desviación estándar (DE) o mediana y rango intercuartílico (RIQ = P25P75) en caso de asimetría de la distribución de la variable.

Para la comparación de variables cualitativas recogidas utilizaremos el test de $\chi^{2}$ o la prueba exacta de Fisher en el caso de que más de $25 \%$ de lo esperado sean menores de cinco.

Para la comparación de variables cuantitativas recogidas del cuestionario general con las subescalas de desgaste profesional como variable cualitativa utilizaremos el test 
ANOVA o de Kruskal-Wallis, previa realización del test de homogeneidad de varianzas de Levene, si las variables siguen una distribución normal en los grupos a comparar y el test no paramétrico de la U de Mann-Whitney si no se ajustan a la distribución normal. El estudio de la normalidad se realizará mediante el test de bondad de ajuste de Kolmogorov-Smirnov. Para todas las pruebas se aceptará un valor de significación de $5 \%(\mathrm{p}<0.05)$.

\section{Resultados}

Inicialmente se incluyeron 32 pacientes con ESA tratados mediante LIF. Se implantaron 16 cajas de PEEK y 16 de titanio. Durante el período postoperatorio tres pacientes fueron excluidos del estudio por pérdida de seguimiento (tres pacientes del grupo PEEK), por lo que se analizaron 29 pacientes que pueden separarse en dos grupos (16 PEEK/13 titanio). La edad media de nuestros pacientes fue 66 años y en su mayoría fueron mujeres (68\%). El dolor irradiado recogido con más frecuencia fue por la pierna derecha (86\%). El EVA lumbar prequirúrgico medio fue de 6.2, el EVA pierna prequirúrgico medio fue de 5.3, el ODI $50.2 \pm 18.9$ y el EQ5D fue 0.52. Ambos grupos presentaban características
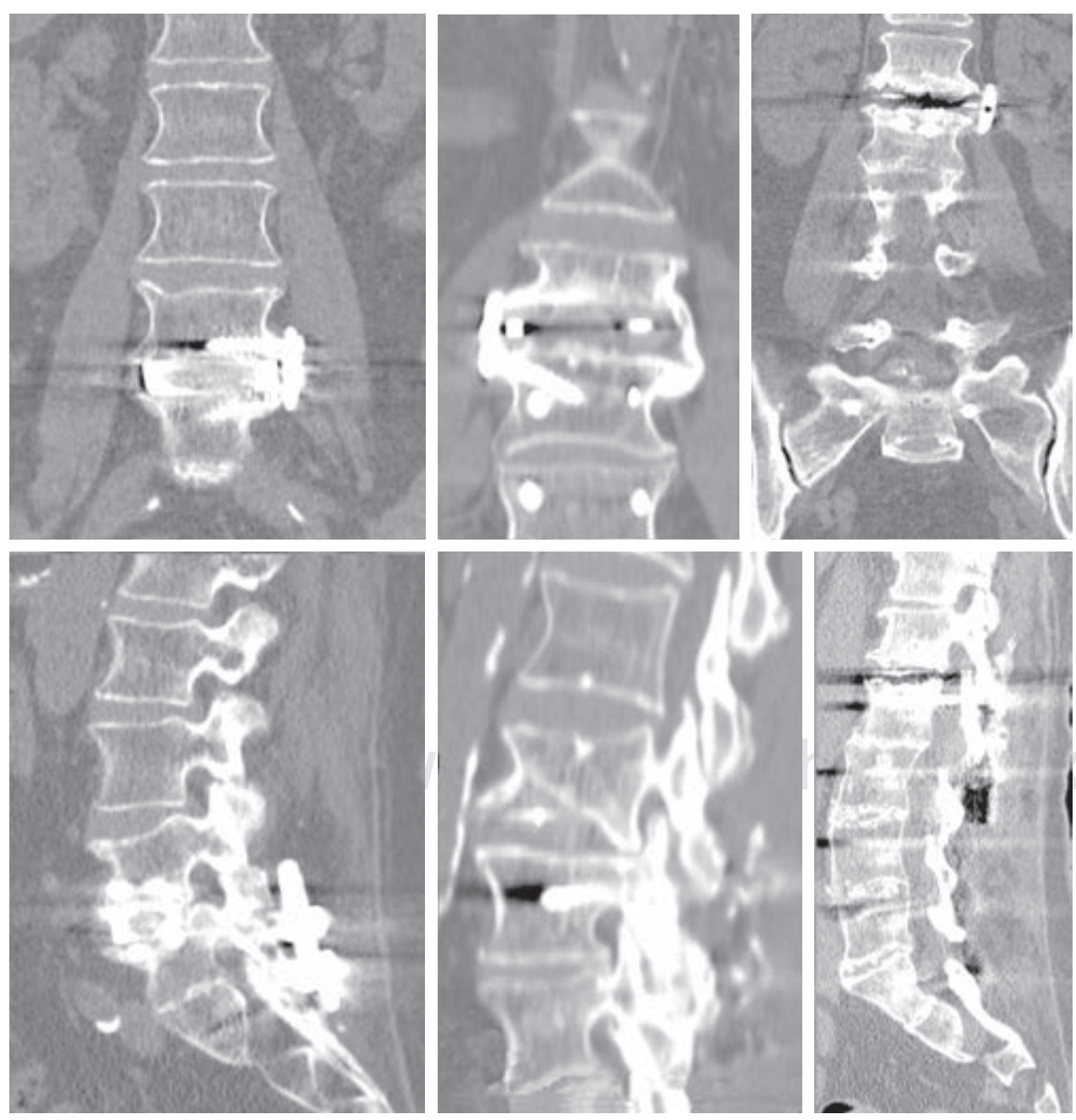

preoperatorias similares, no se encontraron diferencias estadísticamente significativas en la distribución de las variables analizadas en ambos grupos (Tabla 1).

Con respecto a las variables quirúrgicas, el segmento intervenido con más frecuencia fue L3-L4 (62\%) mediante abordaje retroperitoneal derecho $86 \%$. El tamaño del implante que con más frecuencia se utilizó fue $10 \times 22 \times$ $50 \mathrm{~mm}$ (36.8\%). Los implantes empleados presentan una altura de 10, 12 y $14 \mathrm{~mm}$; longitud de 45, 50 y $55 \mathrm{~mm}$ y anchura de 18 y $22 \mathrm{~mm}$. En 66\% de los $\operatorname{casos}^{19}$ se asoció fijación con placa lateral y tornillos (13 PEEK y 6 titanio) y en 10 (34\%) no fue posible implantarla debido a la presencia del nervio femoral en la zona de trabajo (cinco cajas stand alone por grupo). El tiempo de cirugía medio fue 49 minutos, la pérdida de sangre estimada fue de 40 $\mathrm{ml}$. Durante la cirugía se registraron varios cambios en la señal neurofisiológica, cuando aparecieron estas alarmas se realizaron modificaciones técnicas (cambio de colocación del separador o valvas) que permitieron que durante la cirugía las alteraciones desaparecieran y que el control neurofisiológico final fuera normal.

Con respecto al análisis del postoperatorio inmediato (hospitalario), la estancia media fue 2.9 días; $;^{2,3,4} 28 \%$ de
Figura 2:

Ejemplos de los distintos grados de fusión. Imagen de fusión completa a la izquierda, fusión parcial en el centro y falta de fusión a la derecha. 

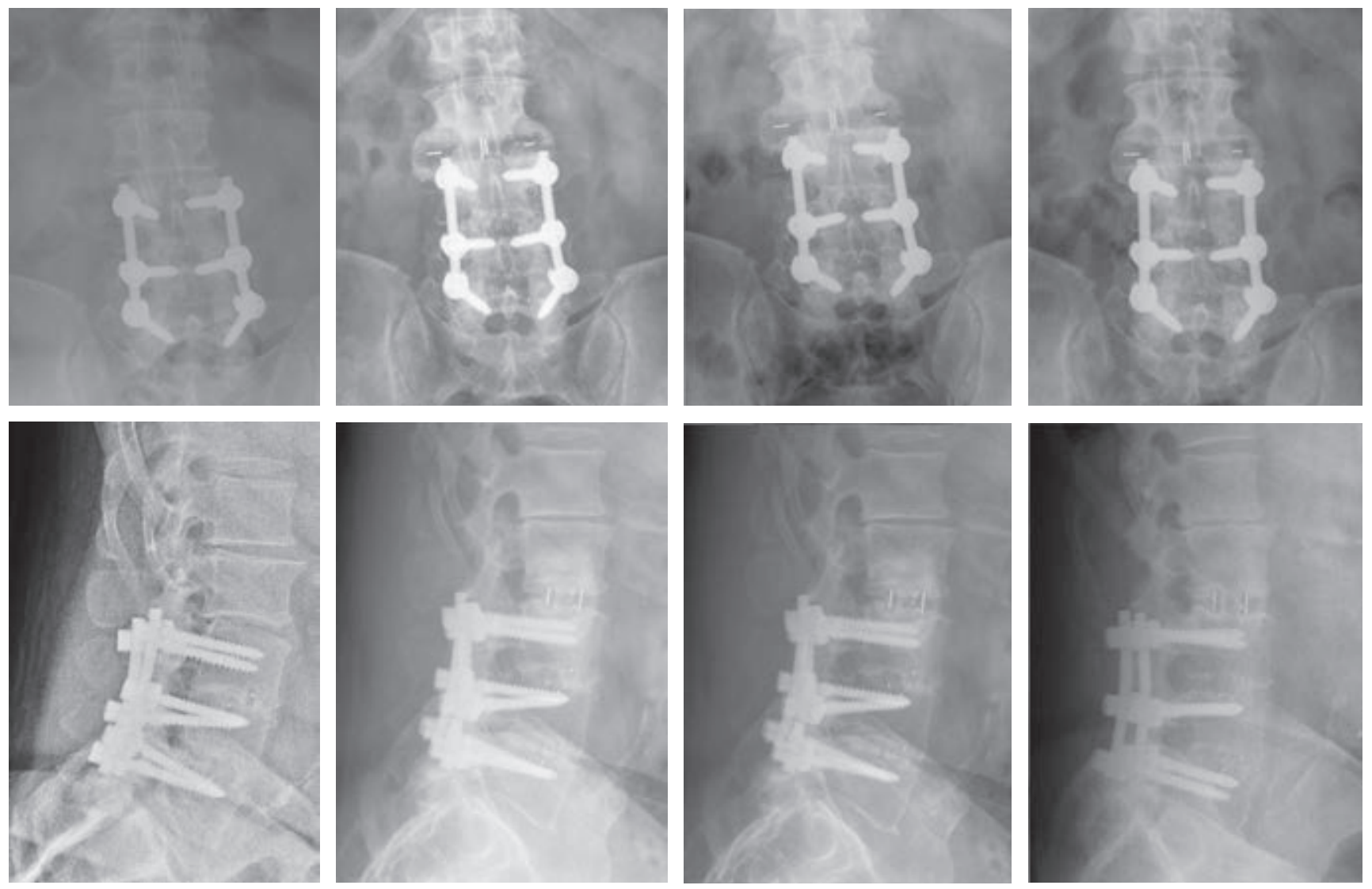

Figura 3: Evolución radiológica de una artrodesis intersomática lateral durante el seguimiento a los tres, seis y 12 meses.

\begin{tabular}{|c|c|c|c|c|}
\hline \multicolumn{5}{|c|}{$\begin{array}{l}\text { Tabla 4: Grado de fusión. } \\
\text { Comparación grado de fusión PEEK vs. titanio. }\end{array}$} \\
\hline Grado & $\begin{array}{l}\text { Global } \\
(\mathrm{N}=29)\end{array}$ & $\begin{array}{l}\text { PEEK } \\
(\mathrm{n}=16)\end{array}$ & $\begin{array}{l}\text { Titanio } \\
(\mathrm{n}=13)\end{array}$ & Significación \\
\hline 1 & 5 & 2 & 3 & 0.798 \\
\hline 2 & 8 & 5 & 3 & 0.325 \\
\hline 3 & 16 & 9 & 7 & 0.568 \\
\hline
\end{tabular}

los pacientes precisaron transfusión de sangre, la necesidad de transfusión de sangre requerida media fue de $0.8 \mathrm{~cm}^{3}$ de hematies (0-2) sin diferencias entre ambos grupos ( $\mathrm{p}=$ $0.15)$. Con respecto a las complicaciones inmediatas menores (precisaron de tratamiento médico para su curación), se encontraron locales menores en $6.9 \%$ : una celulitis de la herida (3.45\%), una plexopatía femoral (3.45\%) (afectación motora del nervio femoral (paresia): un caso de neuroapraxia del femoral ipsilateral al lado de abordaje en el nivel L2-L3. Esta alteración no fue detectada durante el control neurofisiólogo intraoperatorio y se recuperó completamente antes de los seis meses de la intervención con tratamiento médico), ninguna lesión radicular, lesión visceral ni infección). Con respecto a las complicaciones sistémicas menores se encontraron 12 eventos (41.4\%), el más frecuente fue la anemización (siete), infección del tracto urinario (ITU) (dos), íleo paralítico (tres). No se registraron complicaciones inmediatas mayores locales (ninguna lesión de la duramadre, lesión vascular mayor, sangrado retroperitoneal ni infección profunda) ni sistémicas (TVP, TEP fallecimiento). En consecuencia, no se registró ninguna reintervención durante el postoperatorio inmediato. Estas variables presentaron una distribución similar en ambos grupos sin diferencias estadísticamente significativas (Tabla 2). Merece un comentario aparte la presencia de dolor leve-moderado (parestesias) en el tercio proximal del muslo del lado de la vía de abordaje. En nuestra serie estos acontecimientos se observaron en $27.6 \%$ de los casos. ${ }^{7}$ Todos ellos se resolvieron en menos de seis meses. No se han encontrado diferencias estadísticas entre los grupos $(\mathrm{p}=0.563)$.

Desde el punto de vista del resultado radiológico: Se han analizado los cambios de los parámetros prequirúrgicos con los valores al mes, a los seis meses y a los 12 meses (lordosis lumbar (LL), lordosis nivel (LN), Cobb nivel (CN) y la altura del disco intervenido (ADI (AP y LAT)): como hallazgos significativos se evidenció aumento de la LL y LN, disminución del CN y aumento de la ADI en AP y lateral (Tabla 3). Por otra parte, se han comparado los resultados según el tipo de implante: en el inicio el aumento de la LL fue significativamente mayor en el grupo PEEK ( $p=0.029)$. La disminución del 
CN fue mayor en el grupo PEEK a los seis meses ( $\mathrm{p}=$ 0.030). Estos cambios fueron igualándose a lo largo del seguimiento, a los 12 meses no se observaron diferencias significativas en ambos grupos (LL p $=0.367$ y CN p $=$ 0.474) (Tabla 3).

Con respecto a la tasa de fusión, con el TC lumbar al año de la cirugía se encontraron puentes óseos con fusión completa en 16 pacientes (55.2\%), con fusión incompleta en ocho pacientes (27.6\%) y falta de fusión en cinco pacientes $(17.25 \%)$ de la serie, sin evidencias significativas entre los grupos (Figura 3). Considerando la tasa de fusión global (total y parcial) analizada mediante TC es de $84.3 \%{ }^{24}$ y distinguiendo los grupos de estudio: $88 \%$ Ti y $82 \%$ PEEK ( $\mathrm{p}=$ 0.53) (Tabla 4).

Respecto a las complicaciones tardías sistémicas, no se ha recogido ninguna complicación menor ni mayor (ningún caso de fallecimiento).

Como complicaciones tardías locales menores se registró un caso de síndrome facetario lumbar: paciente varón de 47 años con artrodesis lumbar L5-S1 PLIF, hace años que fue intervenido en Diciembre de 2016 realizándose LLIF L4-L5 (titanio), presenta dolor lumbar persistente sin dolor radicular, por lo que en Diciembre de 2017 fue tratado por la unidad de dolor mediante rizólisis.

Como complicaciones tardías locales mayores, cabe señalar que no se encontró ningún caso de fístula de lí- quido cefalorraquídeo o seudomeningocele, ninguna fibrosis perirradicular, hernia abdominal ni infección. Pero se recogieron dos casos de fractura vertebral, una recidiva clínica y una movilización de caja, sin encontrarse diferencias estadísticas entre los grupos. Fractura L4: paciente varón de 85 años con artrodesis lumbar L4-L5 TLIF, hace años que fue intervenido en Noviembre de 2016 realizándose LLIF L3-L4 (PEEK), presenta fractura de L4 en Enero de 2017 (dos meses postquirúgicos) realizándose mediante abordaje bilateral de Wistle retirada de material TLIF previo y artrodesis con tornillos pediculares L2 a L5 (Figura 4). Fractura L3: Paciente mujer 67 años con artrodesis lumbosacra L4-S1 previa que fue intervenida en julio de 2017 realizándose LLIF L3-L4 (titanio), presenta fractura de L3 en Marzo de 2018 que fue intervenida realizándose en un primer tiempo lateral colocación de caja de corrección hiperlordótica. En un segundo tiempo mediante abordaje posterior, extracción de tornillos y barras y se amplía la artrodesis posterior D11S1 (Figura 5). Movilización caja: mujer de 48 años con antecedentes de artrodesis L5-S1 se intervino en Julio de 2017 realizándose LLIF L4-L5 (PEEK) mediante técnica stand-alone, a las cinco semanas de la cirugía comenzó con un importante dolor lumbar irradiado a miembro inferior izquierdo. El estudio de imagen demostró una movilización mayor de $10 \mathrm{~mm}$. Como tratamiento quirúrgico se procedió a la retirada de la caja por la misma vía
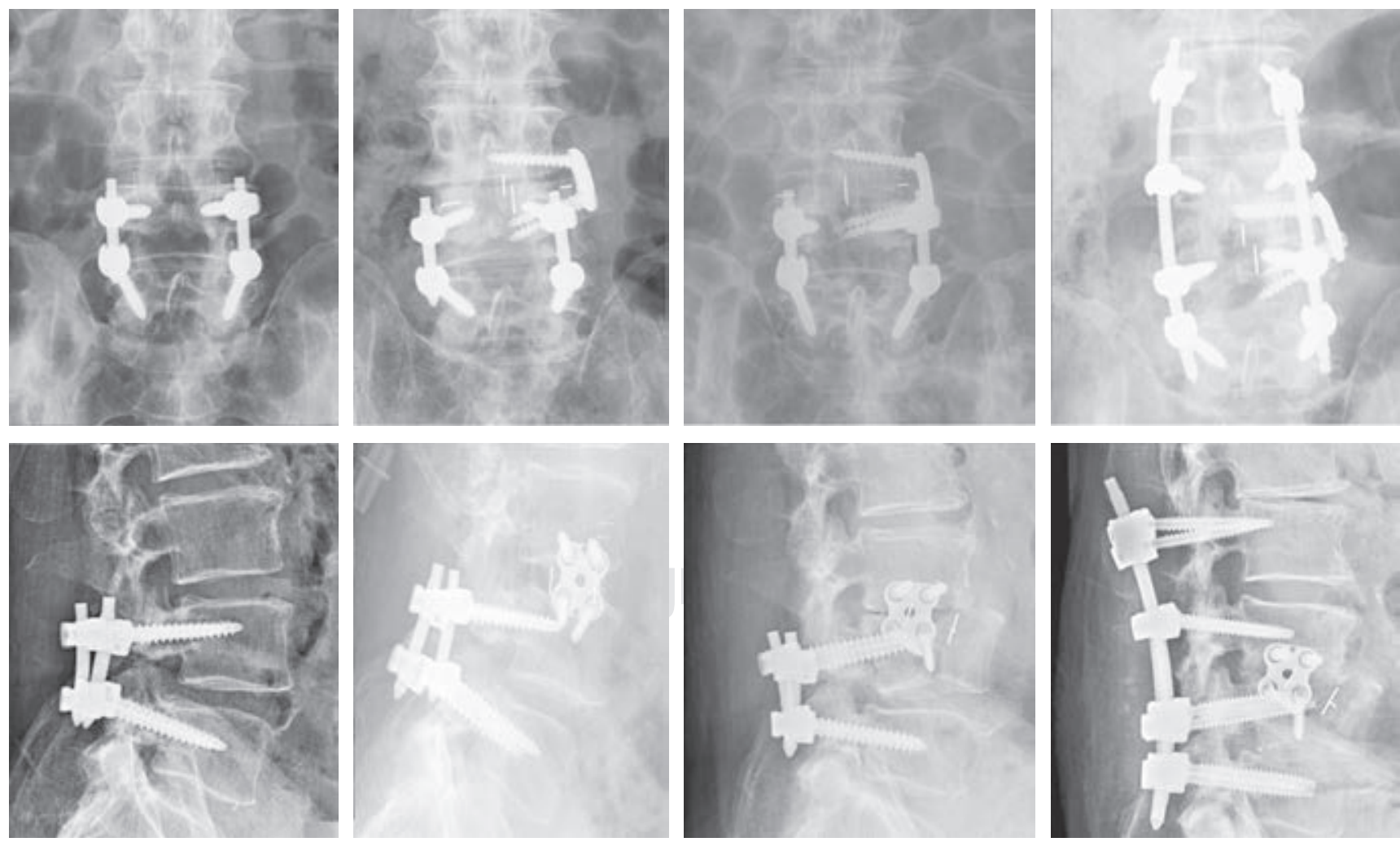

Figura 4: Evolución radiológica de la fractura L4. Imágenes de izquierda a derecha: pre-LLIF, control a los tres meses, complicación y resultado de la revisión. 
transpsoas y ante la imposibilidad de colocar nuevo implante debido a la rotura parcial de la caja y al alto riesgo de lesionar el nervio femoral se decidió rellenar el espacio intersomático mediante injerto con DBM (Figura 6). Recidiva: paciente varón de 82 años con artrodesis lumbar L4-L5 PLIF, hace años que fue intervenido en Julio de 2017 realizándose LLIF L3-L4 (PEEK), presenta reci- diva clínica (dolor lumbar y radicular) en Diciembre de 2017 (cinco meses postquirúrgicos), se realizó mediante abordaje posterior descompresión mediante laminectomía L3. Cuatro pacientes (13.8\%) fueron reintervenidos (con retirada del implante (movilización y una fractura) y se añadió cirugía de fijación posterior (con descompresión en el caso de la recidiva). El estudio comparativo de
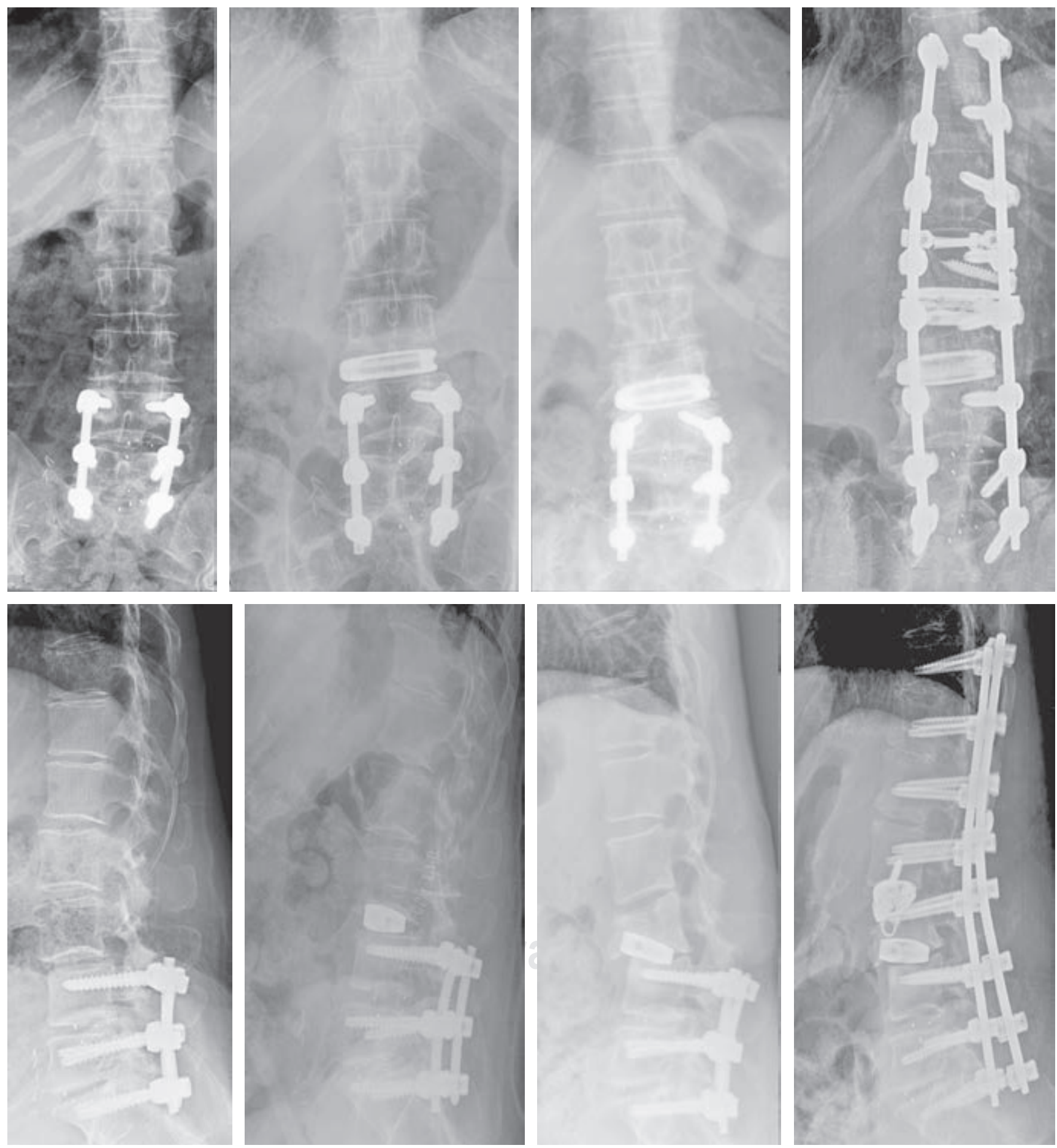

Figura 5: Evolución radiológica de la fractura L3. Imágenes de izquierda a derecha: pre-LLIF, control a los tres meses, complicación y resultado de la revisión. 
las complicaciones no evidenció diferencias respecto al tipo de caja $(\mathrm{p}=0.33) \mathrm{ni}$ con respecto a la necesidad de reintervención $(\mathrm{p}=0.624) \mathrm{ni}$ a la de retirada de la caja ( $\mathrm{p}$ $=0.523$ ) (Tabla 5).

Analizando los resultados clínicos de la serie con respecto al dolor, la puntuación de la escala analógica visual (EVA) lumbar preoperatoria pasó de $6.2 \pm 2.12$ a $4.1 \pm$ $1.71 \mathrm{al}$ año $(\mathrm{p}=0.028)$. La EVA de la pierna descendió de $5.3 \pm 2.26$ en el preoperatorio a $1.9 \pm 1.58$ al año $(\mathrm{p}=$ 0.02). Esta mejoría clínica presentó una distribución similar en ambos grupos sin diferencias estadísticamente significativas. Con respecto a la escala ODI mejoró de 50.2 \pm 18.9 prequirúrgico al final del seguimiento $33.3 \pm 10.2$ $(\mathrm{p}=0025)$. Esta mejoría fue similar en ambos grupos ( $\mathrm{p}$ $=0.181$ ). La puntuación en la escala de calidad de vida EQ5D obtuvo al final del seguimiento un valor de 0.73 , siendo en el inicio de $0.52(p=0.039)$, aunque sin diferencias estadísticamente significativas entre ambos grupos ( $\mathrm{p}$ $=0.293)$ (Tabla 6).

\section{Discusión}

En este estudio clínico prospectivo aleatorizado se han analizado los resultados clínicos y radiológicos, las com- plicaciones y la calidad de vida de los pacientes con ESA intervenidos mediante LLIF. Además, se ha estudiado si hay diferencias distinguiendo el tipo de dispositivos intersomáticos utilizado (Ti/PEEK).

El tratamiento habitual para la ESA es la descompresión posterior y/o extensión de la fusión vertebral,,$^{3,8,9,10,11}$ lo cual muestra una tasa de complicaciones importante con una tasa de morbimortalidad significativa. ${ }^{12,13,14}$ La cirugía LLIF provoca menos daño en las partes blandas en un tiempo quirúrgico más corto y una movilización temprana del paciente, con lo que se ha conseguido menor porcentaje de complicaciones y resultados similares en cuanto a tasa de artrodesis y mejoría clínica. ${ }^{15,16}$

En primer lugar hay que señalar que nuestra serie es similar a las publicadas por otros autores que analizan el tratamiento de la ESA. Las series publicadas van desde series cortas de dos a tres $\operatorname{casos}^{24,25}$ hasta estudios de cerca de 40 pacientes. ${ }^{20,22,26,27}$ Nuestro estudio analiza un mismo procedimiento en un período de tiempo de dos años con un seguimiento medio de 25 meses. Nuestros grupos se formaron mediante la selección del tipo de implante (Ti o PEEK). Con respecto a las características preoperatorias, quirúrgicas y del postoperatorio inmediato no se encontraron diferencias estadísticamente significativas entre los grupos y
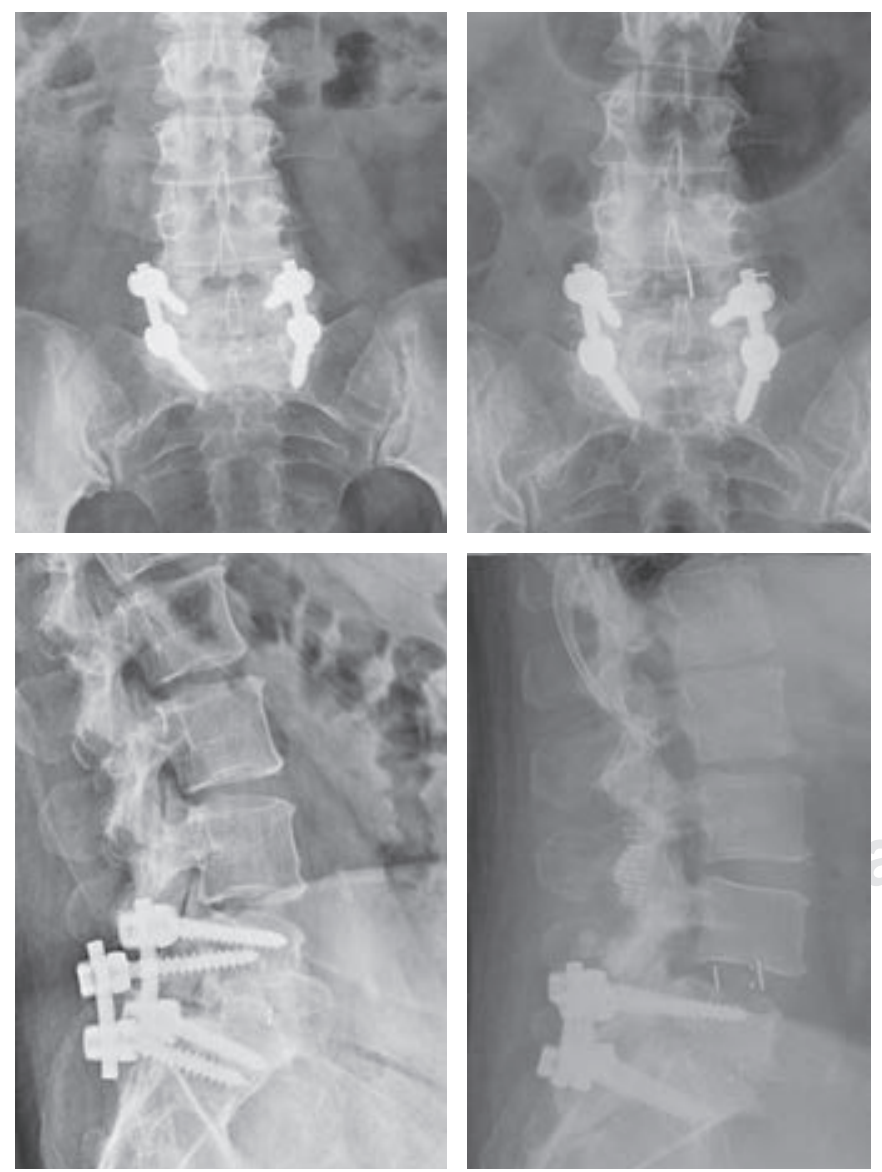
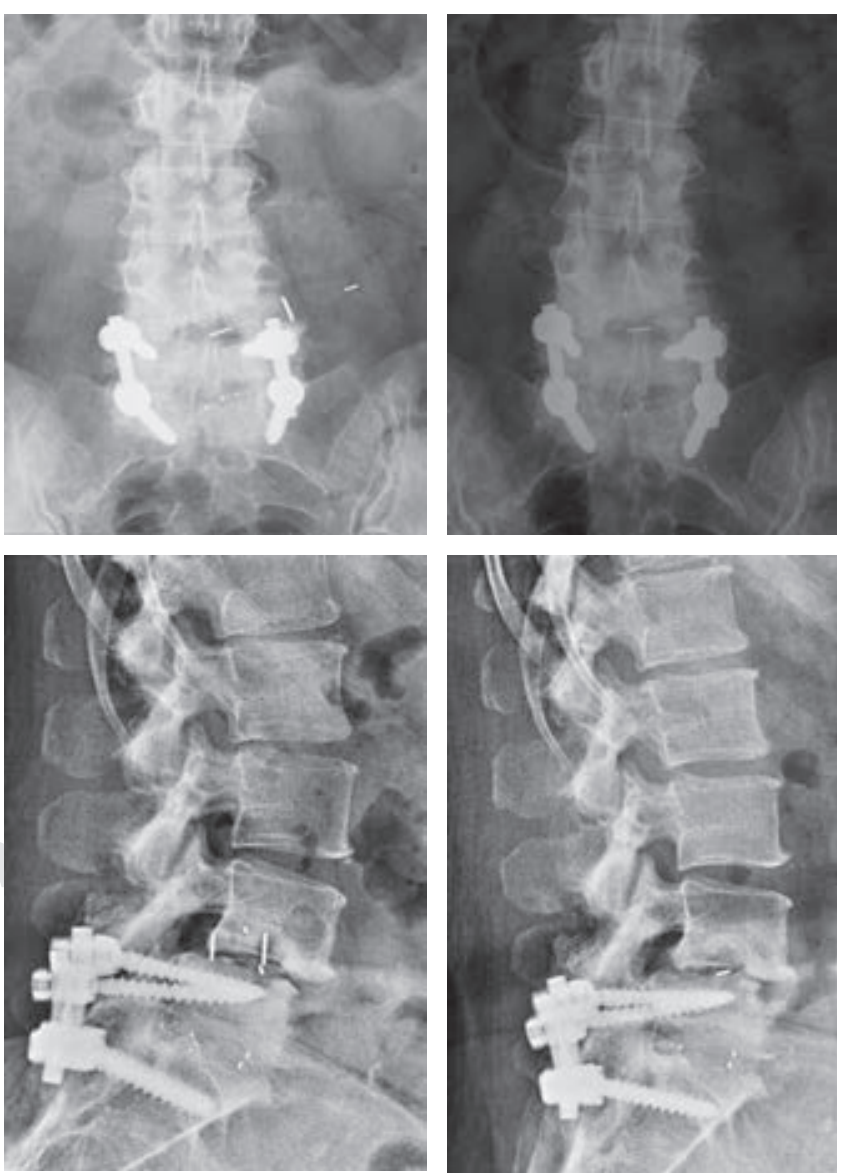

Figura 6: Evolución radiológica de la movilización. Imágenes de izquierda a derecha: pre-LLIF, control a los tres meses, complicación y resultado de la revisión. 
todas las variables fueron similares a las publicaciones recientes. . $4,26,28^{2}$

Con respecto a los resultados clínicos, centrándonos en las publicaciones sobre ESA tratada mediante LLIF, Malham y colaboradores publicaron una mejoría del dolor lumbar de $63 \%{ }^{28}$ y del dolor irradiado por miembro inferior de $56 \%$ al año de la cirugía. Jain y su equipo publicaron en su serie de ESA tratadas con LLIF una mejoría de una EVA lumbar prequirúrgica de $5.1 \pm 3.1$ a $3.3 \pm 2.5(\mathrm{p}=0.049)$ y una mejoría de EVA pierna prequirúrgico de $4 \pm 2.4$ a $1.6 \pm 1.9(\mathrm{p}=0.041) .{ }^{26}$ De forma similar, nuestros pacientes mostraron una mejoría de la puntuación de la escala analógica visual (EVA) lumbar preoperatoria de $6.2 \pm 2.12$ a $4.1 \pm 1.71$ al año $(\mathrm{p}=$ 0.028 ) y la EVA de la pierna descendió de $5.3 \pm 2.26$ en el preoperatorio a $1.9 \pm 1.58$ al año $(\mathrm{p}=0.02)$.

La puntuación en las escalas de discapacidad ODI y de calidad de vida EQ5D mejoraron al final del seguimiento de $50.2 \pm 18.9$ a $33.3 \pm 10.2$ (p=0.025) y de 0.52 a $0.73(\mathrm{p}$ $=0.039$ ), aunque sin diferencias estadísticamente significativas entre ambos grupos (ODI $\mathrm{p}=0.18 \mathrm{EQ5D} \mathrm{p}=0.293$ ). Similar a lo publicado por Jain: ODI mejoría $14.4 \pm 16.9$ (p $=0.028)$ y EQ5D $0.21 \pm 0.23(\mathrm{p}=0.041) \cdot{ }^{26}$ Cabe señalar que esta mejoría clínica fue similar en los dos grupos estudiados, sin diferencias estadísticamente significativas.

Con respecto al análisis de las reintervenciones, la mayoría de los artículos publicados sobre las complicaciones de la cirugía LLIF oscilan alrededor de 2025\%. ${ }^{14,26,27,28}$ Estos porcentajes coinciden con nuestra experiencia: $13.8 \%$ sin diferencias entre los grupos de estudio.

Analizando el resultado radiológico en nuestra serie se evidenció aumento de la LL (5.8 p $=0.00)$ y aumento de la ADI en anteroposterior ( $4.2 \mathrm{~mm} \mathrm{p}=0.023)$ y lateral (4.1 $\mathrm{mm} \mathrm{p}=0.01$ ). Esto es similar a lo publicado por Jain y colaboradores en su serie en la que se recoge un aumento de la lordosis de $5.6 \mathrm{~mm}(\mathrm{p}=0.001)$ y un aumento de la altura del disco de $4.8 \mathrm{~mm}(\mathrm{p}=0.001)$. Comparable con lo publicado en la revisión de Cho y su equipo ${ }^{29}$ con mejoría de la lordosis de 4.7.

Nuestra tasa de fusión analizada mediante TC es de $84.3 \%{ }^{24}$ y distinguiendo los grupos de estudio: $88 \%$ Ti y $82 \%$ PEEK $(p=0.53)$. Similar a las cifras publicadas en la

\begin{tabular}{|ccccc|}
\hline \multicolumn{5}{|c|}{ Tabla 5: Distribución de las complicaciones tardías. } \\
\hline Mayores & $\begin{array}{c}\text { General } \\
(\mathrm{n}=29)\end{array}$ & $\begin{array}{c}\text { Titanio } \\
(\mathrm{n}=13)\end{array}$ & $\begin{array}{c}\text { PEEK } \\
(\mathrm{n}=16)\end{array}$ & Significación \\
Movilización \\
caja (1) \\
$\begin{array}{c}\text { Fractura- } \\
\text { hundimiento (2) } \\
\text { Recidiva (1) } \\
\text { Síndrome } \\
\text { facetario (1) }\end{array}$ & $\begin{array}{c}\text { Fractura- } \\
\text { hundimiento (1) }\end{array}$ & $\begin{array}{c}\text { Movilización } \\
\text { caja (1) } \\
\text { Fractura- } \\
\text { facetario (1) } \\
\text { hundimiento (1) }\end{array}$ & Recidiva (1) & 0.400 \\
& & &
\end{tabular}

Tabla 6: Distribución de la evolución clínica y calidad de vida.

\begin{tabular}{|c|c|c|c|}
\hline & Precirugía & Postcirugía & Significación \\
\hline \multicolumn{4}{|l|}{ EVA Lumbar } \\
\hline Global & $6.2 \pm 2.12$ & $4.1 \pm 1.71$ & 0.028 \\
\hline Titanio & $6.1 \pm 1.78$ & $3.9 \pm 1.58$ & 0.020 \\
\hline PEEK & $6.4 \pm 2.02$ & $4.6 \pm 1.63$ & 0.030 \\
\hline Significación & 0.756 & 0.236 & \\
\hline \multicolumn{4}{|l|}{ EVA Pierna } \\
\hline Global & $5.3 \pm 2.26$ & $1.9 \pm 1.58$ & 0.021 \\
\hline Titanio & $5.1 \pm 2.26$ & $1.9 \pm 2.13$ & 0.023 \\
\hline PEEK & $5.5 \pm 1.65$ & $2.4 \pm 1.56$ & 0.013 \\
\hline Significación & 0.874 & 0.154 & \\
\hline \multicolumn{4}{|l|}{ ODI } \\
\hline Global & $50.2 \pm 18.9$ & $33.3 \pm 10.2$ & 0.025 \\
\hline Titanio & $48.5 \pm 17.4$ & $31.2 \pm 8.8$ & 0.042 \\
\hline PEEK & $50.9 \pm 11.32$ & $34.6 \pm 6.2$ & 0.021 \\
\hline Significación & 0.181 & 0.293 & \\
\hline \multicolumn{4}{|l|}{ EQ5D } \\
\hline Global & $0.52 \pm 0.2$ & $0.73 \pm 0.4$ & 0.039 \\
\hline Titanio & $0.51 \pm 0.7$ & $0.73 \pm 0.8$ & 0.181 \\
\hline PEEK & $0.53 \pm 0.3$ & $0.76 \pm 0.2$ & 0.293 \\
\hline Significación & 0.182 & 0.293 & \\
\hline
\end{tabular}

literatura sobre fusión lumbar intersomática lumbar por Tanida 82.8\% Ti/80.4\% PEEK, Nemoto 100\% Ti/76\% PEEK, Chen 100\% Ti/100\% PEEK, Cabraja 93.2\% Ti 88.1/PEEK, Niu 86.5\% Ti/100 PEEK, Chou 46.5\%/100\% PEEK. ${ }^{20}$ Malham y colaboradores publicaron una tasa de $85 \%$ de fusión completa al año de la cirugía. ${ }^{28}$

Nuestro estudio presenta como limitación principal el número de pacientes estudiados, al tratar de determinar diferencias entre el material del implante serían necesarios estudios con mayor poder estadístico. La ESA es una patología poco frecuente en comparación con la cirugía primaria, por lo que es difícil obtener una muestra mayor. Se necesitan estudios con un seguimiento clínico más amplio para poder estudiar la evolución a largo plazo.

\section{Conclusiones}

- La cirugía LLIF es un método eficaz para el tratamiento de la ESA.

- Se evidencia mejoría clínica y radiológica y en la calidad de vida de los pacientes intervenidos estadísticamente significativa.

- Las complicaciones presentadas son menores que otras vías de abordaje de la ESA y son similares a la literatura publicada; es posible resolverlas y obtener su curación.

- No se han encontrado diferencias estadísticamente significativas entre las variables analizadas en ambos grupos a estudio.

- En el momento actual consideramos la LLIF la técnica ideal para el tratamiento quirúrgico de la ESA. 
Referencias

1. Rajaee SS, Bae HW, Kanim LE, Delamarter RB. Spinal fusion in the United States: analysis of trends from 1998 to 2008. Spine. 2012; 37: 67-76. http://dx.doi.org/10.1097/BRS.0b013e31820cccfb.

2. Domínguez I, Luque R, Noriega M, Rey J, Alia J, Marco-Martínez F. Extreme lateral lumbar interbody fusion. Surgical technique, outcomes and complications after a minimum of one year followup. Rev Esp Cir Ortop Traumatol. 2017; 61(1): 8-18. doi: 10.1016/j. recot.2016.09.001.

3. Lawrence BD, Wang J, Arnold PM, Hermsmeyer J, Norvell DC, Brodke DS, et al. Predicting the risk of adjacent segment pathology after lumbar fusion: a systematic review. Spine (Phila Pa 1976). 2012; 37(22 Suppl): S123-32.

4. Radcliff KE, Kepler CK, Jakoi A, Sidhu GS, Rihn J, Vaccaro AR, et al. Adjacent segment disease in the lumbar spine following different treatment interventions. Spine J. 2013; 13(10): 1339-49.

5. Xia XP, Chen HL, Cheng HB. Prevalence of adjacent segment degeneration after spine surgery: a systematic review and metaanalysis. Spine (Phila Pa 1976). 2013; 38(7): 597-608.

6. Sears WR, Sergides IG, Kazemi N, Smith M, White GJ, Osburg B Incidence and prevalence of surgery at segments adjacent to a previous posterior lumbar arthrodesis. Spine J. 2011; 11: 11-20.

7. Miwa T, Sakaura H, Yamashita T, Suzuki S, Ohwada T. Surgical outcomes of additional posterior lumbar interbody fusion for adjacent segment disease after single-level posterior lumbar interbody fusion. Eur Spine J. 2013; 22(12): 2864-8.

8. Nachanakian A, El Helou A, Alaywan M. The interspinous spacer: a new posterior dynamic stabilization concept for prevention of adjacent segment disease. Adv Orthop. 2013; 2013: 637362.

9. Adogwa O, Parker SL, Shau DN, Mendenhall SK, Devin CJ, Cheng JS, et al. Cost per quality-adjusted life year gained of laminectomy and extension of instrumented fusion for adjacent-segment disease: defining the value of surgical intervention. J Neurosurg Spine. 2012; 16(2): 141-6.

10. Chou D, Dekutoski M, Hermsmeyer J, Norvell DC. The treatment of lumbar adjacent segment pathology after a previous lumbar surgery: a systematic review. Spine (Phila Pa 1976). 2012; 37: S180-8.

11. Parker SL, Shau DN, Mendenhall SK, McGirt MJ. Factors influencing 2-year health care costs in patients undergoing revision lumbar fusion procedures: clinical article. J Neurosurg Spine. 2012; 16(4): 323-8.

12. Smorgick Y, Baker KC, Bachison CC, Herkowitz HN, Montgomery DM, Fischgrund JS. Hidden blood loss during posterior spine fusion surgery. Spine J. 2013; 13(8): 877-81.

13. Parker SL, Mendenhall SK, Shau D, Adogwa O, Cheng JS, Anderson WN, et al. Determination of minimum clinically important difference in pain, disability, and quality of life after extension of fusion for adjacent-segment disease. J Neurosurg Spine. 2012; 16(1): 61-7.

14. Wang MY, Vasudevan R, Mindea SA. Minimally invasive lateral interbody fusion for the treatment of rostral adjacent segment lumbar degenerative stenosis without supplemental pedicle screw fixation: clinical article. J Neurosurg Spine. 2014; 21(6): 861-6.

15. Kepler CK, Sharma AK, Huang RC, et al. Indirect foraminal decompression after lateral transpsoas interbody fusion: clinical article. J Neurosurg Spine. 2012; 16(4): 329-33.

16. Ozgur BM, Aryan HE, Pimenta L, Taylor WR. Extreme lateral interbody fusion (XLIF): a novel surgical technique for anterior lumbar interbody fusion. Spine J. 2006; 6(4): 435-43.
17. Benglis DM, Elhammady MS, Levi AD, Vanni S. Minimally invasive anterolateral approaches for the treatment of back pain and adult degenerative deformity. Neurosurgery. 2008; 63: 191-6.

18. Eck JC, Hodges S, Humphreys SC. Minimally invasive lumbar spinal fusion. J Am Acad Orthop Surg. 2007; 15: 321-9.

19. Uribe JS, Myhre SL, Youssef JA. Preservation or restoration of segmental and regional spinal lordosis using minimally invasive interbody fusion techniques in degenerative lumbar conditions. Spine (Phila Pa 1976). 2016; 41(1): S50-8.

20. Seaman S, Kerezoudis P, Bydon M, Torner JC, Hitchon PW. Titanium vs polyetheretherketone (PEEK) interbody fusion: meta-analysis and review of the literature. J Clin Neurosci. 2017; 44: 23-9. doi: 10.1016/j.jocn.2017.06.062.

21. Chen Y, Wang X, Lu X, Yang L, Yang H, Yuan W, et al. Comparison of titanium and polyetheretherketone (PEEK) cages in the surgical treatment of multilevel cervical spondylotic myelopathy: a prospective, randomized, control study with over 7-year follow-up. Eur Spine J. 2013; 22: 1539-46. doi: 10.1007/s00586-013-2772-y.

22. Nemoto O, Asazuma T, Yato Y, Imabayashi H, Yasuoka H, Fujikawa A. Comparison of fusion rates following transforaminal lumbar interbody fusion using polyetheretherketone cages or titanium cages with transpedicular instrumentation. Eur Spine J. 2014; 23 (10): 21505. doi. 10.1007/s00586-014-3466-9.

23. Chen E, Xu J, Yang S, Zhang Q, Yi H, Liang D, et al. Cage subsidence and fusion rate in extreme lateral interbody fusion with and without fixation. World Neurosurg. 2019; 122: e969-77. doi: 10.1016/j. wneu.2018.10.182.

24. Palejwala SK, Sheen WA, Walter CM, Dunn JH, Baaj AA. Minimally invasive lateral transpsoas interbody fusion using a stand-alone construct for the treatment of adjacent segment disease of the lumbar spine: review of the literature and report of three cases. Clin Neurol Neurosurg. 2014; 124: 90-6. doi: 10.1016/j. clineuro.2014.06.031.

25. Oliveira L, Marchi L, Coutinho E et al. The use of rh-BMP2 in stand alone lateral interbody fusion (XLIF): clinical and radiological results after 24 months follow-up. World Spinal Column J. 2010; 4: 41-6.

26. Jain D, Verma K, Mulvihill J, Mizutani J, Tay B, Burch S, Deviren V. Comparison of stand-alone, transpsoas lateral interbody fusion at L3-4 and cranial vs transforaminal interbody fusion at L3-4 and L4-5 for the treatment of lumbar adjacent segment disease. Int J Spine Surg. 2018; 12(4): 469-74. doi: 10.14444/5056.

27. Rodgers WB, Cox CS, Gerber EJ. Minimally invasive treatment of adjacent segment disease after prior lumbar fusions. Internet $J$ Minimally Invasive Spinal Technol. 2009; 3 (4).

28. Malham GM, Ellis NJ, Parker RM, Seex KA. Clinical outcome and fusion rates after the first 30 extreme lateral interbody fusions. Scientific World Journal. 2012; 2012: 246989.

29. Nakashima H, Kanemura T, Satake K, Ishikawa Y, Ouchida J, Segi $\mathrm{N}$, et al. Changes in sagittal alignment following short-level lumbar interbody fusion: comparison between posterior and lateral lumbar interbody fusions. Asian Spine J. 2019; 13(6): 904-12. doi: 10.31616/ asj.2019.0011.

Financiamiento: Declaro que no existe fuente de financiación. Conflicto de intereses: Declaro que no existe conflicto de intereses durante la elaboración de este trabajo. 